\title{
A prototype of the Laser Guide Stars wavefront sensor for the E-ELT multi-conjugate adaptive optics module
}

\author{
Matteo lombini ${ }^{1,2, a}$, Laura Schreiber ${ }^{2}$, Italo Foppiani ${ }^{2}$, Giovanni Bregoli ${ }^{1}$, Giuseppe Cosentino ${ }^{2}$, \\ Emiliano Diolaiti ${ }^{1}$, Jean-Marc Conan ${ }^{3}$, and Enrico Marchetti ${ }^{4}$ \\ 1 INAF - Osservatorio Astronomico di Bologna, via Ranzani 1, I-40127 Bologna, Italy \\ 2 Universita degli Studi di Bologna - Dipartimento di Astronomia, via Ranzani 1, I-40127 Bologna, \\ Italy \\ 3 ONERA, BP72 - 29 avenue de la Division Leclerc, FR-92322 Chatillon Cedex, France \\ 4 ESO, Karl-Schwarzschild-Strasse 2, D-85748 Garching bei München, Germany
}

\begin{abstract}
The future 42m diameter European Extremely Large Telescope (E-ELT) is foreseen to use Sodium Laser Guide Stars (LGS) in order to increase sky coverage and correction uniformity. The E-ELT MCAO module, MAORY, will use 6 LGSs to measure the Wavefront (WF) aberrations by means of Shack-Hartmann (SH) sensors. However the LGSs are affected by peculiar problems related to the atmospheric Sodium layer. The non-negligible thickness of the layer, for instance, causes the artificial reference source to look elongated, especially in WF sensor sub-apertures at the edge of the pupil. The WF sensor performance are limited by the elongation itself as well as by the fluctuations of the Sodium layer altitude, thickness and density. In particular these fluctuations have dramatic effects on the robustness of the algorithms used for the WF measurement. Moreover the defocus measurement is compromised by a strong non-atmospheric component. Another important aspect is the impact of the low-order aberrations introduced on the LGS images by the MCAO module optics that are optimized for infinite conjugate imaging, while the LGS is at a finite distance. We present in this paper a laboratory prototype that simulates an elongated source that can trace out some LGS features such as the arbitrary profile and altitude variation. In the optical path of the prototype three pupil images are foreseen. High order and low order aberrations can be introduced in the first two pupil planes by means of static screens and a Deformable Mirror (DM) respectively. The WF is then measured by a 40x40 sub-apertures SH Sensor. The main aim of the prototype is to check performance and robustness of WF Sensor algorithms for different illumination conditions, varying aberrations and pixel scale.
\end{abstract}

\section{Introduction}

The construction of the E-ELT[1] is strongly linked to a massive use of adaptive optics to exploit the angular resolution of such a big primary mirror. The need for reference stars for the AO systems leads to a sky coverage problem that can be overcome only by means of artificial sources (Sodium LGSs [2]). Nevertheless using LGSs is not possible to retrieve a tip-tilt measurement and so a combination of Natural Guide Stars (NGSs), for the low order modes measurements [4], and LGSs is needed to obtain a tomographic measurement of the turbulence [3]. Furthermore the finite distance and the thickness of the Sodium layer causes the images of the LGSs to be affected by perspective elongation. On a $40 \mathrm{~m}$ telescope, for instance, the elongation can reach more then 10arcsec. Moreover the fluctuation of the Sodium layer properties (density profile, mean altitude, thickness) drastically decreases the performance of the algorithms used to measure the instantaneous LGS image position in each WF sensor sub-aperture [5]. The scope of the LGS WFS laboratory prototype is to reproduce the relevant aspects of an LGS WFS SH for the E-ELT and, through laboratory tests, evaluate the performance of different centroid algorithms (Weighted center of gravity, correlation and quad-cell) in presence of elongated spots, with realistic Sodium profiles, analyzing the impact of the low-order aberrations and of relevant WFS parameters, as SNR, sampling, sub-aperture size. This study is related to the MAORY [7] design study. In particular we are interested in testing specific technical solutions to be implemented in the module. For instance we want to test the robustness of a possible strategy to deal with the non

\footnotetext{
a e-mail: matteo.lombini@oabo.inaf.it
} the original work is properly cited. 
atmospheric defocus and telescope aberrations which is based on introducing slope measurements offsets at the WF Sensor level. In the next sections we will present the prototype concept, design and the foreseen tests to be carried out. A similar test bench is being developed at the he UVic [6].

\section{Prototype concept}

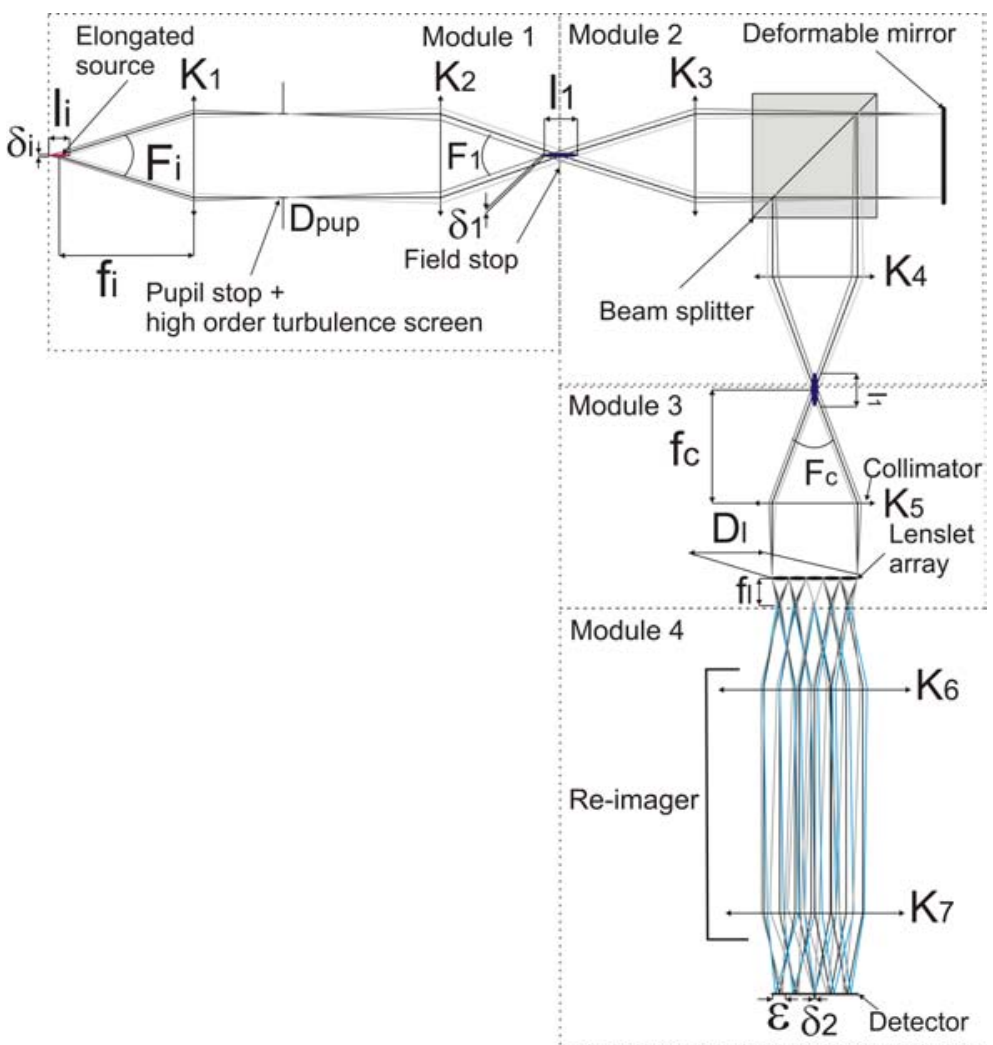

Fig. 1. Sketch of the prototype conceptual design. The system split in three modules can be noticed. The various parameters shown in the figure drive the prototype dimensioning.

The prototype has to generate an elongated light source similar (as seen by the WFS) to an LGS for an E-ELT like telescope. In figure 1 the conceptual scheme of the prototype is presented. The prototype can be divided in four modules. In the first one a point source represented by an optical fiber is moved along the optical axis with a motorized linear stage to simulate the elongated source. After the lens $K_{1}$, a turbulence screen can be placed in the pupil stop plane to introduce high order static aberrations[8] while the lens $K_{2}$ refocuses the image. On the image plane an iris diaphragm acts as field stop setting the system maximum FoV. By reducing the diaphragm aperture the effect of spot truncation can be investigated and the consequences on the algorithms performance evaluated. The second module is a unit magnification afocal system. After the lens $K_{3}$ a beam splitter is placed. The transmitted light (the folded light is lost) is reflected by a DM which can introduce slow varying low order aberrations as would happen in MAORY and can correct the small aberrations introduced by the optical system. The lens $K 4$ refocuses the image. The third module is composed by the collimator $K_{5}$ and a lenslet array having $40 \times 40$ used sub-apertures. After the lenslet array an afocal re-imager (fourth module) is necessary because, due to the small lenslet array focal length, the detector can not be placed immediately after it. Moreover the re-imager increases the plates scale in order to match 
the sub-apertures dimension $(300 \mu \mathrm{m})$ with an integer and odd number of CCD pixels (24 pixels per sub-aperture with $13 \mu \mathrm{m}$ of pixel dimension).

The equation 1 shows the relation between the source cross dimension (fiber core) $\delta_{i}$ and the non elongated side spot FWHM on the detector $\delta_{2}$ :

$$
\delta_{i}=\delta_{2} \cdot M_{r e-i m} \cdot \frac{f_{i}}{f_{c}} \cdot \frac{D_{p u p}}{D_{l}}
$$

where $M_{r e-i m}$ is the re-imager magnification factor. Once the prototype is mounted and the lenses parameters are fixed, the change of plate scale can be done by replacing the fiber with another with different core diameter.

The equation 2 relates the maximum elongation of the spots $e$ on the detector and the source shift along the optical axis $l_{i}$ :

$$
e=l_{i} \cdot \frac{f_{l}}{f_{c}} \cdot \frac{F_{1}}{F_{i}^{2}}
$$

The simulation of the intensity profile of the LGSs[9], related to the varying sodium layer main height and density, is done by inserting an intensity filter before the fiber. The filter is mounted, as the final side of the fiber, on a linear stage. As the stage moves to introduce the elongation on the WFS spots the filter modulates the transmitted light intensity. By changing the filter a different profile can be simulated. The prototype has also the possibility to simulate the side launch of the LGS. Tilting the stage where the output fiber is mounted we can test the non radial elongation pattern of the spots on the SH WFS.

\section{Detailed description}

\subsection{Optical design}

For the detailed optical design (fig. 2) we used only commercial lenses because of the short timescale of this project. In the final optical design the ideal lenses shown in figure 1 has been substituted with group of lenses in order to preserve a good optical quality together with a compact size of the prototype. The optical design was driven by the detector size we had in house (about $1 k x 1 k$ pixels and $13 \mu \mathrm{m}$ pixel size) and by the commercial lenslet arrays pitches and focal lengths that determine the sub-apertures FoV. We chose to work with an $F / 7.5$ focal ratio in order to balance the optical quality with an acceptable dimension of the system. The working wavelength is about $630 \mathrm{~nm}$ to work with almost monochromatic light and moreover with a wavelength similar to the laser light used to align the prototype lenses. The input source is collimated and then focused on the fiber. After the source a passband filter selects the light around 630nm and before the fiber the intensity filter is placed. These filters are plastic screens having a differential transparency along the horizontal axis where the linear stage moves during the prototype measurements. The group $K 1$ that forms the pupil image has been designed with a shorter focal ratio $(F / 4)$ to reduce the fiber movement (eq. 2). The pupil stop diameter is $25 \mathrm{~mm}$ and the DM optical diameter of $15 \mathrm{~mm}$ (14mm used). The lenslet array is formed by $40 \times 40$ used sub-apertures with $300 \mu \mathrm{m}$ pitch and $3.85 \mathrm{~mm}$ focal length.

\subsection{Mechanical design}

The mechanical components are commercial ones, as the linear and tip-tilt stages or the posts, or custom made in Bologna Observatory workshop as the lenslet array mount or the pupil stop. The modules are on separated plates in order to be aligned internally before the whole system integration. The lenses grouped together, as $K_{1}$ in figure 2 that is formed by 4 lenses, are split in two independent mounts (one lens alone and the other three ones in the other mount) that supply translation in the three axes and tip-tilt adjustment. The possibility to change the mounts mutual distance has been foreseen to overcome the likely diversity of lenses actual parameters respect to the catalogue values, preventing 
First conference on Adaptive Optics for Extremely Large Telescopes

Table 1. Relevant parameters of the LGS prototype

\begin{tabular}{lll}
\hline & value & units \\
\hline Wavelength & $0.630 \pm 10$ & $\mathrm{~nm}$ \\
Fiber diameter & 200,300 & $\mu \mathrm{m}$ \\
Pupil stop diameter & 25 & $\mathrm{~mm}$ \\
Field stop diameter & $1-10$ & $\mathrm{~mm}$ \\
DM diameter & 15 & $\mathrm{~mm}$ \\
DM number of actuators & 52 & \\
Lenslet array pitch & 0.3 & $\mathrm{~mm}$ \\
Lenslet array focal length & 3.85 & $\mathrm{~mm}$ \\
Detector pixel size & 13 & $\mu \mathrm{m}$ \\
Detector dimension & $1024 \times 1048$ & pixels \\
Pixel sampling & $1.3,2$ & pixels/FWHM \\
Sub-aperture FoV & 24 & pixels \\
\hline
\end{tabular}
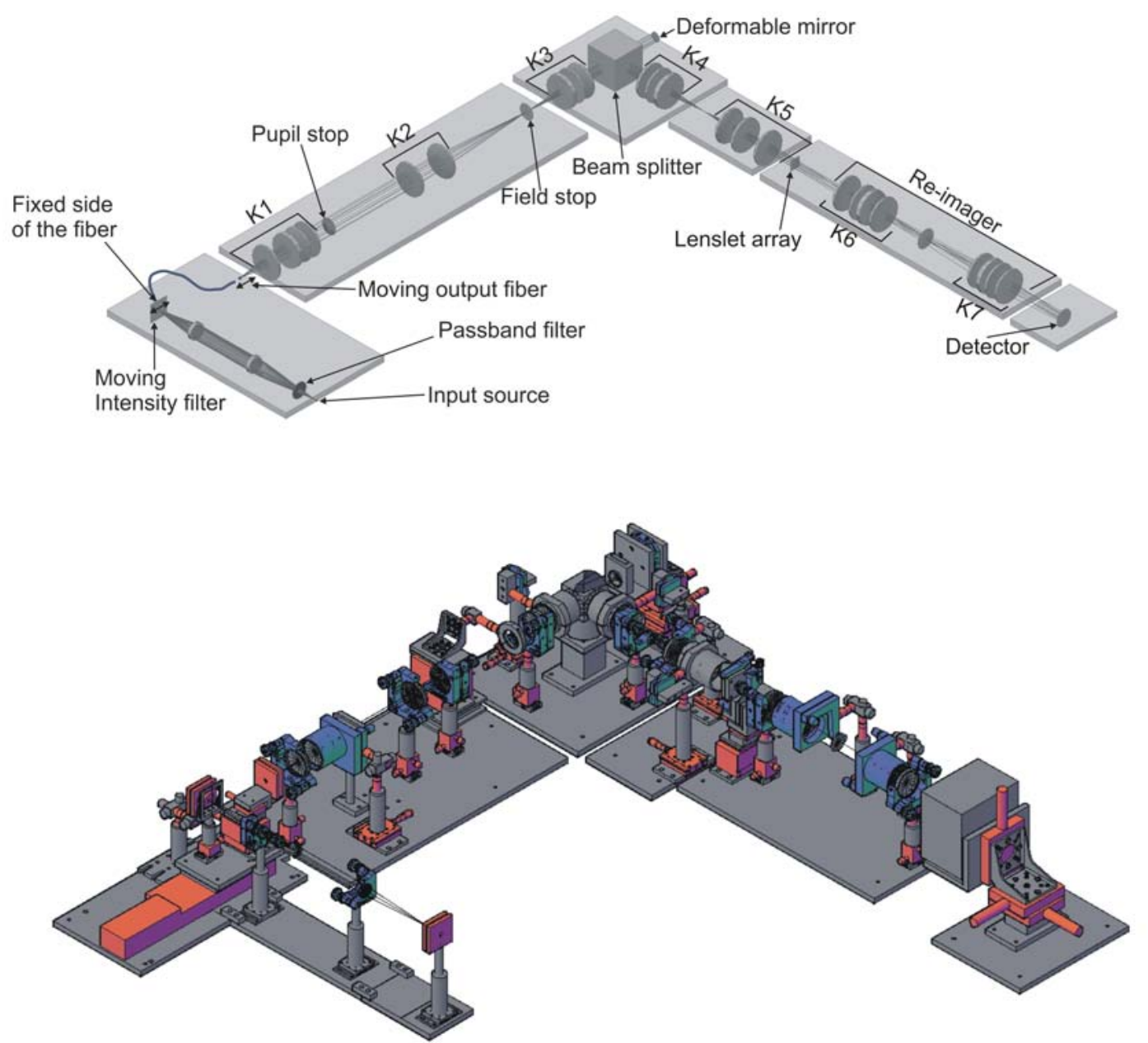

Fig. 2. On the upper figure the optical design of the LGS prototype is presented. On the lower figure we show the mechanical design 
both a decrease of the optical quality and change of the equivalent focal length. Tubes are used to put together the lenses grouped in a single mount and plastic rings of proper thickness regulate their distance.

\subsection{Software}

The software implemented for the prototype tests controls and synchronizes the motorized linear stage, the DM and the Camera. By a graphic interface we can set the parameters of the run. The sequence for acquiring an image is the following:

- Initial parameters setting: light intensity, stage travel, fiber core diameter,DM shape, intensity profile, high order screen

- open detector shutter

- start the linear stage movement

- stop the linear stage

- close detector shutter

- save data

- return stage to initial position

- repeat the sequence ( $\mathrm{n}$ times)

The procedure takes several seconds to take an image. Nevertheless it is very repeatable and it is quite simple to vary single parameters to explore, if necessary, specific critical aspects of the system. The stored images are then analyzed and processed with IDL routines.

\section{Integration and tests}

\subsection{Key components characterization}

All the components used in the prototype must be tested and calibrated before the system integration. For an accurate understanding of the results a deep characterization of the components behavior and thus of error sources must be done. In the following a list of tests that will be done is presented.

- Intensity filters: to test the filter we put it on a linear stage close to the image of a fiber source on a camera and measure the spot intensity variation when moving the stage.

- High order turbulence screens: the WF map is measured with an interferometer

- DM: The relationship signal to actual slope is characterized with an interferometer

- Lenslet Array: measurement of the significant parameters as lens grid regularity, FWHM of the spots and distribution of the scattered light.

- Detector: measurement of RON, Dark Current and Quantum efficiency

\subsection{Prototype alignment}

At the time of writing the status of the project is in the phase of integration of the components and preliminary optical alignment. The strategy for modules integration is to use both an interferometer and a collimated laser beam. The interferometer is used with a reference concave spherical mirror to align the single groups of lenses (for example $K_{6}$ of the re-imager in figure 2) while a plane mirror is mounted in front of the interferometer to position the two groups of a module in the afocal configuration. The laser is used to verity the module magnification. This procedure is likely to be iterative. The modules and the fiber source are then placed in the final position. 


\subsection{Test plan}

The tests planned to characterize the prototype and evaluate the performance of three centroiding algorithms: weighted center of gravity[10], correlation and quad cell.

1. Point source: the fiber is moved along the optical axis to find the position where the smallest defocus term is measured by the WFS. Any residual optical aberration is removed by the DM. This is the prototype alignment final test.

2. Effect of the slope offset for the point source case by properly setting the DM mirror shape

3. Effect of the slope offset for the point source case using different high order screens

4. Tests of points 2 and 3 repeated for a non realistic " rectangular" sodium profile

5. Test of algorithms varying the intensity profile (Gaussian, bi-modal, irregular), SNR, slope offset, elongation, detector binning, high order screens

6. Tests of point 5 with side launch configuration

\section{Acknowledgements}

This work was supported by the European Community (Framework Programme 6, ELT Design Study, contract No 011863; Framework Programme 7, Preparing for the Construction of the European Extremely Large Telescope, contract No INFRA-2007-2.2.1.28) and by the European Southern Observatory (Agreement No 16669/ESO/INS/07/17243/LCO).

\section{References}

1. Gilmozzi, R.; Spyromilio, J.; The European Extremely Large Telescope (E-ELT); The Messenger, volume 127 , page $11 ; 03 / 2007$

2. Hardy, J.; Adaptive optics - A progress review; Active and adaptive optical systems; Proceedings of the Meeting, San Diego, CA, July 22-24, 1991 (A93-39451 15-74), p. 2-17; 1991

3. Ellerbroek, B..; Rigaut, F.; Scaling multiconjugate adaptive optics performance estimates to extremely large telescopes; Proc. SPIE Vol. 4007, p. 1088-1099; 07/2000

4. Flicker, R.; Rigaut, F; Tilt anisoplanatism and PSF retrieval in LGS MCAO using a predictive controller; Proceedings of the Topical Meeting held May 7-10, 2001, Venice, Italy; 00/2002

5. Schreiber, L.; Foppiani, I.; Robert, C.; Diolaiti, E.; Conan, J.-M.; Lombini, M.; Laser guide stars for extremely large telescopes: efficient Shack-Hartmann wavefront sensor design using the weighted centre-of-gravity algorithm; Monthly Notices of the Royal Astronomical Society, Volume 396, Issue 3, pp. 1513-1521;07/2009

6. Thomas, S.; Comparison of centroiding algorithms to optimize Shack Hartmann WFS in the context of ELTs; this conference

7. Diolaiti, E. et al; A preliminary overview of the multi conjugate adaptive optics module for the E-ELT; Proceedings of the SPIE, Volume 7015, pp. 70150U-70150U-11; 2008

8. Thomas, S. ; A simple turbulence simulator for adaptive optics; Proceedings of the SPIE, Volume 5490, pp. 766-773; 2004

9. Pfrommer, T. ; Hickson, P. ; She, C.-Y.; A large-aperture sodium fluorescence lidar with very high resolution for mesopause dynamics and adaptive optics studies; Geophysical Research Letters, Volume 36, Issue 15, CiteID L15831; 08/2009

10. Fusco, T.; Nicolle, M.; Rousset, G.; Michau, V.; Beuzit, J.-L.; Mouillet, D.;Optimization of a Shack-Hartmann-based wavefront sensor for XAO systems; Proceedings of the SPIE, Volume 5490, pp. 1155-1166; 2004 